\title{
Abstracts
}

\section{Franco and Hitler, the Myth of Hendaye 1940}

The Hitler/Franco encounter at Hendaye in October 1940 was a central myth of Francoist propaganda. Allegedly, faced with threats and blandishments to force Spain into war on the Axis side, Franco coolly stood his ground and thereby secured Spanish neutrality. However, there is little evidence that Hitler did threaten Franco. His purpose in travelling to Hendaye, and to Montoire where he met Laval and Pétain, was to compare the relative cost of closer relationships with Spain and Vichy France. Far from cleverly holding off Hitler, Franco was disappointed that the meeting foundered. Germany's need to maintain good relations with Vichy ensured that Hitler could not meet Franco's price for belligerence, the dismemberment of the French North African empire.

\section{War and Social History: Britain and the Home Front During the Second World War}

This article reviews interpretations of the history of British society during the Second World War. Traditionally the Second World War has been viewed as a period of outstanding national unity and social solidarity, and the social arrangements of wartime have been seen as a unique catalyst of administrative 'collectivism' and the growth of the 'welfare state'. More recent historiography has presented a more diffuse picture, emphasising the elements of continuing diversity and conflict in British society during the war period, and the importance of more long-term social trends that were shared by all western European countries.

\section{The Stasi and the East German Revolution of 1989}

Though the GDR's Ministry for State Security (Stasi) had enormous powers in I 989 , it failed adequately to predict or to suppress the Revolution. It encouraged the Communist Party's opposition to reform by providing a distorted picture of society. The Stasi was restricted in its reporting by the ideological blinkers which it shared with the Party. Even during the Revolution, the Stasi believed that popular discontent could be remedied by a vigorous presentation of Party policies. In order 
to understand fully the events of 1989 , it is necessary to view them also through the eyes of the Stasi.

\section{The Origins and Early Development of the Eurobond Market}

This is the transcript of an oral history seminar in which those involved in the early years of the market during the late 1960 described and analysed their experiences. The session began with a short historical overview.

\section{Italian Journals: A User's Guide}

A review of Italian journals dealing with contemporary European history suggests that there have been notable improvements in the standards of Italian historiography in the last decade. This reflects the continued success of Quaderni Storici, the challenge of new periodicals established since the late 1970s, and the response of more established journals to the changed nature of the Italian political contest.

\section{Extraits}

\section{Franco et Hitler. Le mythe de Hendaye 1940}

La rencontre entre Hitler et Franco en octobre 1940 constitue l'un des mythes centraux de la propagande franquiste. Confronté tour à tour aux menaces et séductions destinées à forcer l'Espagne à entrer en guerre aux côtés des forces de l'Axe, Franco s'en serait froidement tenu à ses positions et par là assuré la neutralité de l'Espagne. Il existe cependant peu de preuves que Hitler ait menacé Franco. Le but de son voyage à Hendaye, puis à Montoire où il rencontra Lavel et Pétain, était de comparer le coût relatif de relations plus étroites avec l'Espagne et la France de Vichy. Loin de tenir habilement Hitler à distance, Franco fut en fait déçu de l'issue de la rencontre. Vu la nécessité pour l'Allemagne de maintenir de bonnes relations avec Vichy, Hitler n'était pas pret à payer le prix réclamé par Franco pour son entrée en guerre, à savoir le démembrement de l'empire colonial français en Afrique du Nord.

\section{Guerre et histoire sociale}

Cet article discute les interprétations de l'histoire de la société britannique durant la Seconde Guerre mondiale. La Seconde Guerre mondiale est traditionnellement considérée comme une période d'unité nationale et de solidarité sociale particulierement fortes. On attribue à l'organisation sociale prévalant pendant la guerre un rôle catalyseur dans le 'collectivisme administratif et la croissance de l'Etat providence'. L'historiographie récente présente cependant une image plus contrastée. Elle souligne en particulier le caractère divers et conflictuel de la société britannique, qui se prolonge durant les années de guerre, ainsi que les tendances à long terme que l'on retrouve dans toutes les sociétés occidentales. 


\section{Naissance et développement du marché des eurobonds}

Ce texte est la transcription d'un document d'histoire orale. Les protagonistes des débuts du marché des eurobonds, à la fin des années soixante, y discutent et analysent leurs expériences. $\mathrm{E}$ introduction au séminaire, Kathleen Burk présenta un bref aperçu historique de la question.

\section{La Stasi et la révolution est allemande de 1989}

En dépit de l'énorme pouvoir dont il disposait, le ministère d'Etat pour la sécurité de la République démocratique d'Allemagne (Stasi) fut incapable de prévoir ou de prévenir la révolution. Il encouragea, au sein du parti communiste, l'opposition aux réformes en présentant une image tronquée de la société. La vision de la Stasi était restreinte par les oeillères idéologiques qu'elle paratageait avec le parti communiste. Même durant la révolution, la Stasi pensait que le mécontentement populaire pourrait être désamorcé par une présentation vigoureuse de la politique du parti. Bien comprendre les évènements de 1989 passe par leur examen à travers les yeux de la Stasi.

\section{Revues italiennes}

La principale conclusion que se dégage d'un compte rendu critique des revues italiennes consacrées à l'histoire contemporaine de l'Europe est que le niveau de l'historiographie italienne s'est sensiblement amélioré au cours de la dernière décennie. Ceci se reflète en particulier dans le succès de Quaderni Storici, le défi représenté par les nouvelles revues établies depuis la fin des années I970, et la réponse des revues plus anciennes au nouveau climat politique italien.

\section{Kurzfassungen}

\section{Franco und Hitler: Die Legende von Hendaye}

Das Treffen Hitlers mit Franco Oktober 1940 ist zu einer der zentralen Mythen der Franco-Propaganda geworden. Der Legende nach hat Franco trotz Drohung und Schmeichelei an seiner Position festgehalten, dass Spanien sich in den Krieg auf Seite der Achsenmächte nicht einmischen sollte. In dieser Weise wurde die Neutralität seines Landes garantiert. Es gibt jedoch keine Beweise dafür, dass Hitler Franco gedroht hat. Er war nach Hendaye und auch nach Montoire, wo er Laval und Petain traf, angereist, um die relativen Kosten näherer Zusammenarbeit mit Spanien oder mit der Vichyregierung Frankreichs zu vergleichen. Es war nicht Hitler sondern Franco der entaüscht wurde, weil die Notwendigkeit auf Seite Deutschlands gute Beziehungen zur Vichyregierung aufrechtzuerhalten bedeutete, dass Hitler nicht bereit war, den Preis der spanischen Unterstützung im Krieg, namentlich die Zergliederung des französichen nordafrikanischen Reichs, zu bezahlen. 


\section{Krieg und Sozialgeschichte}

Dieser Beitrag beschäftigt sich mit den verschiedenen Interpretationen der Entwicklung der britischen Gesellschaft während des Zweiten Weltkriegs. Normalerweise wird diese Periode der britischen Geschichte als eine Periode ausserordentlicher nationaler Einigkeit angesehen. Die sozialen Intiativen der Kriegszeit werden als einen einzigartigen Anreiz zum 'Verwaltungskollektivismus' und zur Entwicklung des Wohlfahrtsstaates gewertet. Neulich ist die Geschichtsschreibung nuancierter geworden. Jetzt wird Elemente der Meinungsvielfalt und des Sozialkonfliktes stärker betont, wie auch die Wichtigkeit langfristiger sozialer Prozesse, die alle europäische Länder gemeinsam hatten.

\section{Das Ministerium für Staatsicherheit der DDR (Stasi) und die ostdeutsche Revolution von 1989}

Obwohl das Ministerium 1989 über enorme Kräfte verfügte, war es ihm nicht gelungen die Revolution weder vorherzusagen noch zu unterdrücken. Es versuchte die Opposition innerhalb der Kommunistischen Partei zu Reformmassnahmen zu bewegen durch die Präsentation eines verzerrtes Gesellschaftsbildes. Die Staasiberichte waren nicht imstande ein realistisches Bild der Situation zu verbreiten aufgrund der Tatsache, dass das Ministerium genau dieselbe ideologische Scheuklappen trug als die Partei. Sogar während der Revolution glaubte es, dass die Volksunzufriedenheit durch eine energische Präsentation der Parteipolitik zu beheben war. Um die Ereignisse des Jahres I 989 voll und ganz zu verstehen muss man sie auch mit Stasiaugen ansehen.

\section{Die Ursprung und Frühentwicklung des Eurobondmarkts}

Es liegt ein Protokoll eines Geschichtsseminars, wobei die Leute, die sich an den Frühjahren des Marktes Ende der sechsziger Jahre beteiligten, ihre Erfahrungen beschreiben und analysieren. Die Sitzung wurde mit einem kurzem historischem Überblick eingefürt.

\section{Italiensche Zeitschriften}

Dieser Überblick der italienischen Zeitschriften, die sich mit der europäischen Zeitgeschichte beschäftigen, deutet darauf an, dass im letzten Jahrzehnt die italienische Geschichtsschreibung sich viel verbessert hat. Diese Tatsache ist eine Folge des andauernden Erfolgs der Zeitschrift Quaderni Storici, der Herausforderung der seit den späten siebzig Jahren erschienenen neuen Zeitschriften und der Antwort der etablierten Zeitschriften auf die geänderte Situation des politischen Kampfes in Italien. 